\title{
Etiology Diagnosis and Management of Radial Nerve Entrapment
}

\author{
Neeraj Vij ${ }^{1}{ }^{1,}{ }^{*}$, Hayley Kiernan ${ }^{1}$, Sam Miller-Gutierrez ${ }^{2}$, Veena Agusala ${ }^{3}$, Alan David Kaye ${ }^{4}$, Farnad \\ Imani (iD) ${ }^{5}$, Behrooz Zaman (iD ${ }^{5, * *}$, Giustino Varrassi (iD) ${ }^{6}$, Omar Viswanath (iD ${ }^{1,4,7,8}$ and Ivan Urits (iD $)^{4,9}$ \\ ${ }^{1}$ University of Arizona College of Medicine-Phoenix, Phoenix, AZ, USA \\ ${ }^{2}$ Department of Medicine and Biomedical Engineering, Sarver Heart Center, University of Arizona, Tucson, AZ, USA \\ ${ }^{3}$ Texas Tech University Health Sciences Center, School of Medicine in Lubbock, TX, USA \\ ${ }^{4}$ Louisiana State University Health Shreveport, Department of Anesthesiology, Shreveport, LA, USA \\ ${ }_{5}^{5}$ Pain Research Center, Department of Anesthesiology and Pain Medicine, Iran University of Medical Sciences, Tehran, Iran \\ ${ }^{6}$ Paolo Procacci Foundation, Via Tacito 7, Roma, Italy \\ ${ }^{7}$ Creighton University School of Medicine, Department of Anesthesiology, Omaha, NE, USA \\ ${ }^{8}$ Valley Anesthesiology and Pain Consultants - Envision Physician Services, Phoenix, AZ, USA \\ ${ }^{9}$ Southcoast Health, Southcoast Health Physicians Group Pain Medicine, Wareham, MA, USA \\ "Corresponding author: University of Arizona College of Medicine-Phoenix, Phoenix, AZ, USA. Email: neerajvij@email.arizona.edu \\ ** Corresponding author: Pain Research Center, Department of Anesthesiology and Pain Medicine, Iran University of Medical Sciences, Tehran, Iran. Email: \\ behroozzaman@gmail.com
}

Received 2021 January 10; Accepted 2021 January 17.

\section{Abstract}

Context: The anatomy of the radial nerve is prone to entrapment, each with different symptomology. Compression of entrapment of the radial nerve can occur near the radiocapitellar joint, the spiral groove, the arcade of Frohse, the tendon of the extensor carpi radialis brevis (ECRB), and at the radial tunnel. Those who require repetitive motions are at increased risk of peripheral neuropathy syndromes, including repetitive pronation and supination, trauma, or systemic disease; however, t the influence of all risk factors is not well understood. Depending on the location of entrapment, radial nerve entrapment syndrome presents different symptoms. It may include both a motor component and a sensory component. The motor component includes a dropped arm, and the sensory component can include pain and paresthesia in the distribution of the radial nerve that resolves with rest and exacerbates by repetitive pronation and supination.

Evidence Acquisition: Diagnostic evaluation for radial nerve entrapment, apart from clinical symptoms and physical exam, includes electromyography, nerve conduction studies, ultrasonography, and magnetic resonance imaging. Conservative management for radial nerve entrapment includes oral anti-inflammatory medications, activity modification, and splinting. Some recently performed studies mentioned promising minimally invasive techniques, including corticosteroid injections, peripheral nerve stimulation, and pulsed radiofrequency.

Results: When minimally invasive techniques fail, open or endoscopic surgery can be performed to release the nerve

Conclusions: Endoscopic surgery has the benefit of decreasing incision size and reducing time to functional recovery.

Keywords: Radial Nerve, Entrapment, Neuropathy, Minimally Invasive, Injections, Surgical Treatment

\section{Context}

The radial nerve is the largest branch of the brachial plexus, which traverses posteriorly around the humerus, bifurcating at the radiocapitellar joint, forming the superficial and deep branches. This anatomy provides an opportunity for radial nerve entrapment resulting in peripheral neuropathy of the arm, forearm, and hand. Entrapment, or compression of radial nerve neuropathy, can be categorized as compressive and non-compressive. Compressive neuropathies are more prevalent and can occur under a variety of circumstances, most commonly from repetitive activities, either recreational or occupational (1). The extent of nerve injury depends on the intensity of the mechanical and the temporal compression. Subsequent injury can be categorized based on increasing severity (i.e., neuropraxia, axonotmesis, and neurotmesis) (2). Symptoms may not manifest at the time of the initial insult or injury. Radial nerve entrapment symptomatology can be misperceived as musculoskeletal pathologies, which contributes to underdiagnosis of radial nerve injury and delayed treatment (1). Physical examination is not sufficient to diagnose entrapment syndromes (3). Utilizing a plurality of tools available to diagnose radial nerve injuries, such as magnetic resonance imaging (MRI), ultrasonography (US), and neural tension testing, can reduce the incidence of misdiagnosis. The current study aimed to, first, provide a summary of the 
current state of knowledge regarding the etiology and diagnosis of the radial nerve entrapment and then updating the surgical and non-surgical management of this condition.

\subsection{Risk Factors}

Radial nerve entrapment, compressive and noncompressive neuropathies are attributed to different etiologies. Non-compression related insults are less common but can present with an abrupt onset of symptoms. These include traumatic injuries, such as humeral fractures and lacerations, or systemic disease (4). Non-compression related insults can progress to the encapsulation of the radial nerve. Callus formation around the initial insult region can act to compresses the nerve, which results in the manifestation of symptoms (2). Primary compression related neuropathies are far more common. Overuse, particularly repetitive supination and pronation motions, cause an increased risk of nerve compression. For instance, excessive typing (occupational) and tennis (recreational). The latter can lead to compression at the lateral epicondyle; the deep radial nerve travels laterally to the lateral epicondyle, then under the arcade of Frohse, which predisposes the nerve to excessive mechanical stress with overuse (5).

\subsection{Clinical Presentation}

Presenting symptoms are dependent largely on the location and the type of insult. Patients may be asymptomatic as long as nerve conduction is not hindered, leading to under-recognized pathology. Persistent nerve compression can result in transient neuropraxia and chronic axonotmesis, leading to decreased motor function, sensation, or increased pain. Transient disruption of afferent nerve conduction may result in mild symptoms that can be alleviated with minimal intervention and rest (2-6). Radial nerve entrapment can result in distal upper limb pain and paresthesias, which can be exacerbated with repeated pronation of the forearm (1). Chronic and consistent compression may cause Axonotmesis, which initiates demyelination. In turn, symptoms of pain and weakness become more persistent and prominent with the progression of this neuropathy. Progressive demyelination can result in Wallerian degeneration, a notable clinical marker for electrodiagnosis (4). Axonotmesis results in impaired motor function; symptoms commonly include loss of or impaired mobility in the brachioradialis, supinator muscles, and the extensor carpi radialis longus and brevis muscles (7). Finally, the most severe radial nerve neuropathy is neurotmesis, which is the complete transection of the radial nerve caused by blunt trauma or surgical insult.

\subsection{Diagnosis}

Several tests are available for diagnosing compression neuropathies that each has its strengths and weaknesses, which are dependent on the desired endpoint and the region of the test. The diagnostic can be made via imaging and non-imaging techniques (8). The latter includes electrodiagnostic studies, including electromyography (EMG) and nerve conduction studies (NCS). EMG is fairly limited in breadth; however, it is a useful technique for ruling out pathologies that mimic radial neuropathies (4). NCS is particularly useful in identifying demyelination at the spiral groove, though additional techniques are required to identify the lesion at a higher level of resolution (4). EMG and NCS can be used for diagnosing and grading peripheral nerve injuries; however, they cannot often identify the anatomical cause of chronic injuries (9). The primary modes of imaging are ultrasonography (US) and magnetic resonance imaging (MRI). These are more powerful tools that can directly identify the lesion. The US has improved in resolution over time; however, the remained inherent limitations are due to operator dependency, which leads to varied testing results. The introduction of broadband high-frequency transducers elevates the US to serve as a powerful diagnostic tool in specific locations of radial neuropathies, such as at the spiral groove (10). An advantage of MR imaging over the US is the higher soft-tissue contrast while reducing user variability (11). Additionally, resolving images have improved with high-resolution MR imaging, which allows the distinction between normal nerves and those with pathologies (12). In summation, more than one diagnostic test would likely be required to accurately diagnose most entrapment radial nerve entrapment syndromes.

\subsection{Anatomy}

The radial nerve is a branch of the brachial plexus that serves to supply the upper limbs and is composed of nerve fibers $\mathrm{C} 5$ to T1. The radial nerve has two branches at the radiocapitellar joint: the superficial radial branch and the posterior interosseous nerves (PIN) branch. The superficial branch supplies mostly sensory innervation, and the PIN supplies mostly motor innervation (4). Motor innervation of the radial nerve includes the triceps brachii, anconeus, supinator, brachioradialis, abductor pollicis longus, and the wrist and hand extensor muscles (13). Sensory innervation includes the posterior arm and forearm, the lateral aspect of the dorsal hand, the dorsal thumb, and dorsal lateral two and a half digits (14).

The radial nerve can be entrapped at multiple locations along its course through the upper extremity. The PIN travels along the radial neck, then pierces the supinator muscle, which is the most common site of entrapment (15). The 
PIN further divides into four terminal branches, which can all become compressed at their locations within what is termed the radial tunnel. These sites include the radial head, the Leash of Henry (recurrent radial vessels), the arcade of Frohse, and the tendon of the extensor carpi radialis brevis (15). Repetitive pronation and supination are also associated with radial nerve entrapment (16).

\subsection{Pathophysiology}

Radial nerve injury typically occurs due to compression, entrapment, traction, or direct trauma to the nerve, which causes a local inflammatory response with swelling and edema. Compressive or entrapment of the nerve results in microvascular damage to nerves and their myelin sheaths. Prolonged compression results in an inflammatory response and subsequent fibrosis and demyelination, which can worsen the mechanical effects of nerve compression (17). As during this process the myelination losts, an interruption in the speed of axonal signaling can occur, which ultimately leads to a partial or complete block of action potentials through the affected nerve segment (17). Persistent entrapment of the radial nerve, with the combination of these factors, may lead to axonal degeneration and long-term nerve damage.

In mild cases, this compression of the nerve causes no permanent damage, and the nerve may fully recover. As the length of entrapment increases, permanent damage can ensue, causing persistent deficits (15). Multiple classification systems are used to categorize nerve injury grading. The sunderland classification is a popular grading system that rates nerve injuries as 1 st - 5th degree depending on the amount of physical damage to the nerve itself, as well as the degree of damage to the myelin sheath and axon (18). The time required for nerve recovery will vary depending on the degree of damage sustained, as remyelination may only weeks, while axonal regrowth can take much longer (19).

\section{Evidence Acquisition}

\subsection{Medical Management}

Conservative management remains to be the first line for radial nerve entrapment syndrome. This includes oral anti-inflammatory medications, activity modification to avoid provocative activities, and splinting $(2,18$, 19)However, there is minimal evidence in the literature regarding the success of these therapies. Many articles do confirm this and state that although conservative medical management, as well as activity modification and splinting, may be the first line, further treatments with betterknown efficacies are often needed (20). Carter et al. (2015), in an evidence-based review, argued that medical management should be trialed for a minimum of six weeks, with consecutive uninterrupted treatment before progressing to other treatments (21).

\subsection{Minimally Invasive Techniques}

Several minimally invasive techniques for radial nerve entrapment have been described in the literature. Some discussion around lidocaine infusion for refractory chronic pain exists (22), though further investigation of this therapy with regards to pirifmoris syndrome is needed. Corticosteroid injections are amongst the first methods tried. Several authors do mention the potential for corticosteroid injections $(2,15,21,23,24)$. However, to date, no large-scale study has investigated the efficacy of corticosteroid injections for radial nerve entrapment.

The peripheral nerve stimulation with an implanted device is a relatively new and proven effective treatment modality (12, 25-27). This device is inserted under US guidance. A few studies pointed out the potential for this technique in several peripheral neuropathies, including radial nerve entrapment. Huntoon et al. (2009) described the success of this technique in several peripheral neuropathies, including the radial nerve. Five (out of six) patients who underwent permanent PNS system implantation (after a successful trial) had significant pain relief at the last follow-up (25). Though this paper demonstrated the potential for this therapy, it is important to note that $1 / 8$ of patients did have an infection after implantation at the implantation site.

Radiofrequency has significantly improved in the past few years (28). Oh et al. (2016) described pulsed radiofrequency for the radial nerve in refractory lateral epicondylitis (tennis elbow) in two patients. Though, a large-scale randomized scale trial is needed to determine, pulsed radiofrequency may show some promise in addition to the above mentioned minimally invasive techniques before considering as surgical techniques.

\subsection{Surgical Techniques}

Surgical interventions for nerve entrapment syndromes continue to evolve in pursuit of smaller incisions, shorter recovery times, and utilization of surgical planes that minimize the risk of iatrogenic injury $(29,30)$. Surgical treatment should only be pursued after the trial of at least 3 - 6 months of non-operative options $(21,23)$. Ebrahimzadeh et al. (2015) argued that surgical options demonstrated a success rate of up to $92 \%$ and $95 \%$ for release of the PIN only and PIN/SBRN, respectively (23). Several open surgical techniques are described. A larger surgical field allows for fewer iatrogenic injuries; however, 
it involves an incision and scar. Additionally, some complications have been recorded, and the risk of opioid use disorder in surgical patients is of some concern (6)

Endoscopic techniques are less invasive and are proved to reduce post-operative recovery time. They have a high success rate and are desirable by their reduction in postoperative recovery time and smaller incisions than open techniques (31). Contrary to what is reported by prior research, the risk of developing a pain syndrome at the retinaculum transection or the heel of the hand ("pillar pain"), the efficacy in terms of recovery beyond 6 months, and the reduction of symptoms were comparable in both the open and endoscopic surgical options.

During surgery, non-contact laser Doppler flowmetry has been identified as a simple way to study the changes of perfusion to the nerve, in real-time, during the release procedures for an entrapped nerve (32).

\section{Results}

Thus, it has the potential to decrease incision length necessary for peripheral nerve surgeries, including radial interventions with no increase in perioperative morbidity. Doppler flowmetry can be used in conjunction with an endoscopic release to minimize incision length and time to functional recovery (33).

\section{Conclusions}

Radial nerve entrapment can be attributed to compressive and non-compressive neuropathies. These can occur due to several different etiologies, including primary compression from overuse, repetitive supination, and pronation motions. Persistent nerve compression can result in transient neuropraxia and chronic axonotmesis, leading to decreased motor function, sensation, or increased pain. Therefore, once clinical symptoms are observed, apt diagnosis and prudent intervention are of crucial importance.

A wide array of diagnostic tools are available to clinicians, including ultrasonography, magnetic resonance imaging, electromyography, and nerve conduction studies. Selecting the proper method can support early diagnosis and treatment.

Surgical interventions should be considered when medical management and other conservative measures fail after 3 - 6 months of trial. Endoscopic and open release of the nerve at the entrapped site are among the available options. An endoscopic release may be preferred as it is associated with the shortest recovery times and smallest incisions. Ultrasound-guided intervention, as well as the use of doppler flowmetry to intraoperatively monitor blood flow, are promising new methods for improved surgical intervention.

\section{Footnotes}

Authors' Contribution: Study concept and design; NV and IU; Acquisition of data: NV and IU; Analysis and Interpretation of data: NV, SMG, VA, HK, AK, FI, BZ, GV, OV, IU; Drafting of the Manuscript: NV, SMG, VA, HK, AK, FI, BZ, GV, $\mathrm{OV}, \mathrm{IU}$; Critical revision of the manuscript for important intellectual content: NV, SMG, VA, HK, AK, FI, BZ, GV, OV, IU; Statistical Analysis: none; Administrative, technical, and material support: NV and IU; Study supervision: NV and IU.

Conflict of Interests: The authors declare no conflict of interest.

Funding/Support: None.

\section{References}

1. Neal SJ, Fields KB. Peripheral nerve entrapment and injury in the upper extremity. Am Fam Physician. 2010;81(2):147-55.

2. Latef TJ, Bilal M, Vetter M, Iwanaga J, Oskouian RJ, Tubbs RS. Injury of the radial nerve in the arm: A review. Cureus. 2018;10(2). e2199. doi: 10.7759/cureus.2199. [PubMed: 29666777]. [PubMed Central: PMC5902095].

3. Ong C, Nallamshetty HS, Nazarian LN, Rekant MS, Mandel S. Sonographic diagnosis of posterior interosseous nerve entrapment syndrome. Radiol Case Rep. 2007;2(1):1-4. doi: 10.2484/rcr.v2i1.67. [PubMed: 27303450]. [PubMed Central: PMC4891580].

4. Wang LH, Weiss MD. Anatomical, clinical, and electrodiagnostic features of radial neuropathies. Phys Med Rehabil Clin NAm. 2013;24(1):3347. doi: 10.1016/j.pmr.2012.08.018. [PubMed: 23177029].

5. Ekstrom RA, Holden K. Examination of and intervention for a patient with chronic lateral elbow pain with signs of nerve entrapment. Phys Ther. 2002;82(11):1077-86. doi:10.1093/ptj/82.11.1077.

6. Malik KM, Imani F, Beckerly R, Chovatiya R. Risk of opioid use disorder from exposure to opioids in the perioperative period: A systematic review. Anesth Pain Med. 2020;10(1). e101339. doi:10.5812/aapm.101339. [PubMed: 32337175]. [PubMed Central: PMC7158240].

7. Doughty CT, Bowley MP. Entrapment neuropathies of the upper extremity. Med Clin North Am. 2019;103(2):357-70. doi: 10.1016/j.mcna.2018.10.012. [PubMed: 30704687].

8. Nejati P, Sartaj E, Imani F, Moeineddin R, Nejati L, Safavi M. Accuracy of the diagnostic tests of sacroiliac joint dysfunction.J Chiropr Med. 2020;19(1):28-37. doi: 10.1016/j.jcm.2019.12.002. [PubMed: 33192189]. [PubMed Central: PMC7646135].

9. Jengojan S, Kovar F, Breitenseher J, Weber M, Prayer D, Kasprian G. Acute radial nerve entrapment at the spiral groove: detection by DTI-based neurography. Eur Radiol. 2015;25(6):1678-83. doi: 10.1007/s00330-014-3562-6. [PubMed: 25576227].

10. Martinoli C, Bianchi S, Pugliese F, Bacigalupo L, Gauglio C, Valle $M$, et al. Sonography of entrapment neuropathies in the upper limb (wrist excluded). J Clin Ultrasound. 2004;32(9):438-50. doi: 10.1002/jcu.20067. [PubMed:15558622].

11. Kim S, Choi JY, Huh YM, Song HT, Lee SA, Kim SM, et al. Role of mag netic resonance imaging in entrapment and compressive neuropathy. What, where, and how to see the peripheral nerves on the musculoskeletal magnetic resonance image: part 2. Upper extremity. Eur Radiol. 2007;17(2):509-22. doi: 10.1007/s00330-006-0180-y. [PubMed: 16572333]. 
12. Ray WZ, Mahan MA, Guo D, Guo D, Kliot M. An update on addressing important peripheral nerve problems: challenges and potential solutions. Acta Neurochir(Wien). 2017;159(9):1765-73. doi: 10.1007/s00701017-3203-3. [PubMed: 28500566].

13. Jefferson-Falardeau J, Houle S. Chiropractic management of a patient with radial nerve entrapment symptoms: A case study.J Chiropr Med. 2019;18(4):327-34. doi: 10.1016/j.jcm.2019.07.003. [PubMed: 32952479]. [PubMed Central: PMC7486464].

14. Barral J, Croibier A. Anatomy and physiology of the peripheral nervous system. 1st ed. New York: Elsevier Health Sciences; 2007. doi: 10.1016/b978-0-443-10307-0.50005-7.

15. Thurston A. Radial tunnel syndrome. Orthop.Trauma.2013;27(6):403-8.

16. Olewnik L, Podgorski M, Polguj M, Wysiadecki G, Topol M. Anatomical variations of the pronator teres muscle in a Central European population and its clinical significance. Anat Sci Int. 2018;93(2):299-306. doi: 10.1007/s12565-017-0413-y. [PubMed: 28849397]. [PubMed Central: PMC5797209].

17. Rempel DM, Diao E. Entrapment neuropathies: pathophysiology and pathogenesis. J Electromyogr Kinesiol. 2004;14(1):71-5. doi: 10.1016/j.jelekin.2003.09.009. [PubMed: 14759752].

18. Chhabra A,Ahlawat S, Belzberg A, Andreseik G. Peripheral nerve injury grading simplified on MR neurography: As referenced to Seddon and Sunderland classifications. Indian J Radiol Imaging. 2014;24(3):217-24. doi: 10.4103/0971-3026.137025. [PubMed: 25114384]. [PubMed Central: PMC4126136].

19. Boyajian-O'Neill LA, McClain RL, Coleman MK, Thomas PP. Diagnosis and management of piriformis syndrome: an osteopathic approach. J Am Osteopath Assoc. 2008;108(11):657-64. doi: 10.7556/jaoa.2008.108.11.657. [PubMed: 19011229].

20. Naam NH, Nemani S. Radial tunnel syndrome. Orthop Clin North Am. 2012;43(4):529-36. doi:10.1016/j.ocl.2012.07.022. [PubMed: 23026469].

21. Carter GT, Weiss MD. Diagnosis and treatment of work-related proximal median and radial nerve entrapment. Phys Med Rehabil Clin N Am. 2015;26(3):539-49. doi: 10.1016/j.pmr.2015.04.001. [PubMed: 26231964].

22. Tully J, Jung JW, Patel A, Tukan A, Kandula S, Doan A, et al. Utilization of intravenous lidocaine infusion for the treatment of refractory chronic pain. Anesth Pain Med. 2020;10(6). e112290. doi: 10.5812/aapm.112290.

23. Moradi A, Ebrahimzadeh MH, Jupiter JB. Radial tunnel syndrome, di- agnostic and treatment dilemma. Arch Bone Jt Surg. 2015;3(3):156-62. [PubMed: 26213698]. [PubMed Central: PMC4507067].

24. Imani F, Varrassi G. Ketamine as adjuvant for acute pain management. Anesth Pain Med. 2019;9(6). e100178. doi: 10.5812/aapm.100178. [PubMed: 32280623]. [PubMed Central: PMC7119219].

25. Huntoon MA, Burgher AH. Ultrasound-guided permanent implantation of peripheral nerve stimulation (PNS) system for neuropathic pain of the extremities: Original cases and outcomes. Pain Med. 2009;10(8):1369-77. doi: 10.1111/j.1526-4637.2009.00745.x. [PubMed: 20021597].

26. Urits I, Schwartz R, Smoots D, Koop L, Veeravelli S, Orhurhu V, et al. Peripheral Neuromodulation for the Management of Headache. Anesth Pain Med. 2020;10(6). e110515. doi: 10.5812/aapm.110515.

27. Divizia M, Germani G, Urti I, Imani F, Varrassi G, et al. Endoscopic neuromodulation of suprascapular nerve in chronic shoulder pain: A case Report. Anesth Pain Med. 2020;10(2). e103624. doi: 10.5812/aapm.103624. [PubMed: 32754436]. [PubMed Central: PMC7352948].

28. Sluijter ME, Imani F. Evolution and mode of action of pulsed radiofrequency. Anesth Pain Med. 2013;2(4):139-41. doi: 10.5812/aapm.10213. [PubMed: 24223349]. [PubMed Central: PMC3821144].

29. Orhurhu V, Orman S, Peck J, Urits I, Orhurhu MS, Jones MR, et al. Carpal tunnel release surgery: A systematic review of open and endoscopic approaches. Anesth Pain Med. 2020;10(6). e112291. doi: 10.5812/aapm.112291.

30. Vij N, Traube B, Bisht R, Singleton I, Cornett EM, Kaye AD, et al. An update on treatment modalities for ulnar nerve entrapment: a literature review. Anesth Pain Med. 2020;10(6). e112070. doi: 10.5812/aapm.112070.

31. Leclere FM, Bignion D, Franz T, Mathys L, Vogelin E. Endoscopically assisted nerve decompression of rare nerve compression syndromes at the upper extremity. Arch Orthop Trauma Surg. 2013;133(4):575-82. doi: 10.1007/s00402-012-1668-3. [PubMed: 23417113].

32. Soejima O, Iida H, Naito M. Measurement of median nerve blood flow during carpal tunnel release with laser Doppler flowmetry. Minim Invasive Neurosurg. 2001;44(4):202-4. doi: 10.1055/s-2001-19938. [PubMed: 11830778].

33. Ducic I, Endara M, Al-Attar A, Quadri H. Minimally invasive peripheral nerve surgery: a short scar technique. Microsurgery. 2010;30(8):622-6. doi: 10.1002/micr.20810. [PubMed: 20842707]. 